\title{
Silencing circRNA_001937 may inhibit cutaneous squamous cell carcinoma proliferation and induce apoptosis by preventing the sponging of the miRNA-597-3p/FOSL2 pathway
}

\author{
LING GAO $^{1 *}$, HONG-JUAN JIN ${ }^{2 *}$, DUO ZHANG ${ }^{2}$ and QUAN LIN ${ }^{2}$ \\ Departments of ${ }^{1}$ Radiation Oncology and ${ }^{2}$ Plastic Surgery, The First Hospital of Jilin University, \\ Changchun, Jilin 130021, P.R. China
}

Received July 20, 2019; Accepted December 30, 2019

DOI: $10.3892 /$ ijmm.2020.4723

\begin{abstract}
Circular RNAs (circRNAs) are reported to be aberrantly expressed and perform different functions in numerous types of tumor; however, their expression levels in cutaneous squamous cell carcinoma (CSCC) remain largely unclear. Thus, the purpose of the present study was to investigate the function of circRNA_001937 in CSCC. Differential circRNA expression profiles of CSCC were analyzed using the Arraystar Human circRNAs chip and reverse transcription-quantitative PCR (RT-qPCR); and the effects of circRNA_001937 on cell behavior, in particular its regulation over the microRNA (miRNA)-597-3p/Fos-related antigen 2 (FOSL2) pathway, was investigated using a dual-luciferase reporter assay, and verified using RT-qPCR and western blotting. circRNA_001937 expression levels were significantly increased in CSCC tissues and cell lines compared with the corresponding adjacent tissues and control cells $(\mathrm{P}<0.05)$. The genetic silencing of circRNA_001937 with small interfering RNA significantly inhibited cell proliferation, and induced cell apoptosis $(\mathrm{P}<0.05)$. circRNA_001937 was observed to directly bind to miRNA-597-3p and serve as a sponge, which indirectly increased the expression levels of FOSL2, a miRNA-597-3p target gene. In conclusion, circRNA_001937 expression was increased in CSCC and silencing circRNA_001937 gene expression may inhibit CSCC progression by sponging the miRNA-597-3p/FOSL2 pathway.
\end{abstract}

Correspondence to: Dr Quan Lin, Department of Plastic Surgery, The First Hospital of Jilin University, 71 Xinmin Street, Changchun, Jilin 130021, P.R. China

E-mail: linquanmail@yeah.net

*Contributed equally

Key words: cutaneous squamous cell carcinoma, circular RNA_001937, microRNA-597-3p, Fos-related antigen 2, proliferation

\section{Introduction}

Cutaneous squamous cell carcinoma (CSCC) is one of the most common skin malignancies, accounting for $25-35 \%$ of all skin cancer types (1). The extent of tumor infiltration and metastasis greatly influences the clinical stage and prognosis of the disease; and although numerous treatment options are available for CSCC, including surgical excision, radiotherapy, photodynamic therapy and topical drug treatment, the prognosis of invasive and metastatic CSCC remains relatively poor and is associated with substantial levels of mortality (2). Furthermore, the incidence of CSCC continues to increase, which is largely due to the increased prevalence of risk factors including older populations, immunosuppression, chronic sun exposure and sensitivity to sunlight or ultraviolet radiation $(1,3)$.

Circular RNAs (circRNAs) are a newly identified group of non-coding RNAs that lack 5'-caps and 3'-tails (4), which leaves them resistant to exonuclease- or ribonuclease-mediated degradation and permits their stable expression in numerous types of organisms. A number of studies have reported that circRNA expression levels are significantly increased in various types of tumor, where they serve as important molecules for tumor metastasis and recurrence (5). circRNA may competitively bind to microRNA (miRNA) response elements to inhibit miRNA expression or function, which ultimately affects target genes $(6,7)$. Fos-related antigen 2 (FOSL2) is a member of the activator protein 1 (AP-1) transcription factor family, which includes the various isoforms of Fos and Jun (8-10). Previous studies have demonstrated that FOSL2 is abnormally expressed in numerous different types of tumor, where it serves important functions in cell adhesion, migration, invasion, metastasis and proliferation (11-12).

The aim of the present study was to investigate the function of circRNA_001937 in CSCC. In the present study, differential circRNA expression profiles of CSCC were analyzed using the Arraystar Human circRNAs chip and verified by RT-qPCR. In addition, the effects of circRNA on cell behavior, in particular its regulation of the miRNA-mRNA axis, were also investigated. 


\section{Materials and methods}

Patient samples. The present study was approved by the Ethics Committee of the First Hospital of Jilin University (Jilin, China) and written informed consent was obtained from all patients. Three pairs of CSCC tissues and corresponding adjacent tissues were collected from the Department of Plastic Surgery at the First Hospital of Jilin University between September 2015 and November 2018. The clinicopathological features are shown in detail in Table SI. All specimens were confirmed by clinical and pathological diagnosis.

Cell lines. The human CSCC cell lines A431 and SCL-1, and the human immortal keratinocyte cell line $\mathrm{HaCaT}$, were purchased from Guangzhou Genio Biotechnology Co., Ltd.

Reagents. circRNA_001937, miRNA-597-3p and FOSL2 mRNA primer sequences were purchased from Kangcheng Co., Ltd. Anti-FOSL2 primary antibody (1:500; cat. no. H00116173-B01P) and anti-GAPDH primary antibody (1:1,000; cat. no. R2655) were purchased from Sigma-Aldrich (Merck KGaA). RPMI-1640 medium, fetal bovine serum (FBS), crystal violet, Annexin V fluorescein isothiocyanate (FITC)/propidium iodide (PI) Cell Apoptosis Detection kit (cat. no. sc-4252 AK), MTT assays, Transwell plates, Matrigel and dimethyl sulfoxide were purchased from Santa Cruz Biotechnology, Inc.

Profiling of circRNA expression. The Arraystar Human circRNAs chip (Arraystar Inc.) was used to analyze the expression of circRNAs in the CSCC tissues and corresponding adjacent tissues. Total RNA was extracted from the samples using an RNeasy Mini kit (cat. no. 74104; Qiagen $\mathrm{GmbH}$ ), and the RNA was analyzed on the circRNAs chips. The expression levels were analyzed and quantified by Kangcheng Co., Ltd.

Reverse transcription-quantitative PCR (RT-qPCR). Total RNA was extracted from A431 and SCL-1 cells using TRIzol ${ }^{\circledast}$ reagent (Thermo Fisher Scientific, Inc.). Total RNA was reverse transcribed into cDNA at room temperature using $\operatorname{TaqMan}^{\mathrm{TM}}$ reverse transcription reagents (cat. no. 4304134; Thermo Fisher Scientific, Inc.). qPCR was subsequently performed using the iScript ${ }^{\mathrm{TM}}$ cDNA Synthesis kit (cat. no. 1708890; Bio-Rad Laboratories, Inc.). The following thermocycling conditions were used for the qPCR: 40 cycles at $94^{\circ} \mathrm{C}$ for $15 \mathrm{sec} ; 20$ cycles at $55^{\circ} \mathrm{C}$ for $30 \mathrm{sec}$; and 20 cycles at $70^{\circ} \mathrm{C}$ for $30 \mathrm{sec}$. The following primers were used: circRNA_001937 forward, 5'-TGAAGAACAGCTCTC TGGCTG-3' and reverse, 5'-GCCCACTTAATCAGGGTC AGGT-3'; miRNA-597-3p forward, 5'-CGGAATTCATCT CAAGCCAAC-3' and reverse, 5'-CGGGATCCCTTCATT CAAGGTCAATG-3'; FOSL2 forward, 5'-GAGAGGAAC AAGCTGGCTGC-3' and reverse, 5'-GCTTCTCCTTCT CCTTCTGC-3'; U6 (control for miRNA) forward, 5'-TTT AGGGCTTCGATACT-3' and reverse, 5'-TCTGCTGCA GCACA-3'; and GAPDH (control for circRNA and mRNA) forward, 5'-GGTCCTGTTGTTTA-3' and reverse, 5'-TGC TCATTCCCTC-3'. Expression levels were quantified using the $2^{-\triangle \Delta C q}$ method (13) and the relative expression levels of target RNAs were normalized to the loading control U6.
Cell transfection. Small interfering RNA (siRNA/si) targeting circRNA_001937 (si-circRNA_001937) and the negative control (si-NC) labeled with green fluorescent protein, miRNA-597-3p mimic, miRNA-597-3p inhibitor and the NC were synthesized by Kangcheng Co., Ltd. The sequence of si-circRNA_001937 was 5'-GGCAGCACATGTCAGGC-3' and the sequence of si-NC was 5'-TCTTTAGGGGTGTGC GTAGG-3'. The quantity of siRNA transfected was $50 \mathrm{nM}$. A431 and SCL-1 cells (1x10\% $/$ well) were transfected using Lipofectamine ${ }^{\circledR} 2000$ reagent (Thermo Fisher Scientific, Inc.) according to the manufacturer's protocol. Cells were transfected for $48 \mathrm{~h}$ prior to subsequent experimentation.

MTT assay. Following transfection, A431 and SCL-1 cells in the logarithmic growth phase were seeded at a density of $1 \times 10^{5} /$ well into 96 -well culture plates. Following $0,12,24$ or $48 \mathrm{~h}$ of culture, $20 \mu \mathrm{l}$ MTT solution was added/well for $6 \mathrm{~h}$ to determine cell proliferation. Following the MTT incubation, cells were washed with phosphate buffered saline (PBS) and the purple formazan crystals were dissolved using $100 \mu \mathrm{l}$ dimethyl sulfoxide/well. Proliferation was subsequently analyzed at a wavelength of $490 \mathrm{~nm}$ using a microtiter plate reader.

Colony formation assay. Following transfection, A431 and SCL-1 cells ( $1 \times 10^{4} /$ well) in the logarithmic growth phase were plated into 6-well culture plates and cultured for 2 weeks at $37^{\circ} \mathrm{C}$. Following incubation, the cells were washed twice with PBS, fixed with $2 \%$ paraformaldehyde at $37^{\circ} \mathrm{C}$ for $30 \mathrm{~min}$ and subsequently stained with $0.5 \%$ crystal violet at $37^{\circ} \mathrm{C}$ for $15 \mathrm{~min}$. Colonies were visualized using a Nikon electron microscope (magnification, x100; Nikon Corporation) and analyzed using ImageJ version 1.47 software (National Institutes of Health).

Matrigel invasion assay. Following transfection, A431 and SCL-1 cells (1×105/well) in the logarithmic growth phase were plated in the upper chambers of Transwell plates with Matrigel and fibronectin was also added to the upper chamber. RPMI-1640 medium supplemented with 20\% FBS was plated in the lower chambers. Following incubation at $37^{\circ} \mathrm{C}$ for $24 \mathrm{~h}$, the lower chamber cells were fixed with $2 \%$ paraformaldehyde at $37^{\circ} \mathrm{C}$ for $30 \mathrm{~min}$ and stained with $0.5 \%$ crystal violet at $37^{\circ} \mathrm{C}$ for $15 \mathrm{~min}$. Stained A431 and SCL-1 cells were visualized using a confocal microscope (magnification, $\mathrm{x} 100$ ) and ImageJ version 1.47 software was used to quantify the number of invasive cells.

Flow cytometric analysis of apoptosis. Cell apoptosis was performed using the Annexin V-FITC/PI apoptosis detection kit, according to the manufacturer's protocol. Following transfection, A431 and SCL-1 cells in the logarithmic growth phase were seeded into 96-well culture plates and were subsequently incubated with Annexin V-FITC and PI solution at $37^{\circ} \mathrm{C}$ for $20 \mathrm{~min}$ in the dark. Apoptotic cells were visualized using a BD FACSCalibur flow cytometer (BD Biosciences).

Dual-luciferase reporter assay. A431 and SCL-1 cells in the logarithmic growth phase were digested and seeded into 24-well culture plates. Subsequently, the A431 and SCL-1 cells 

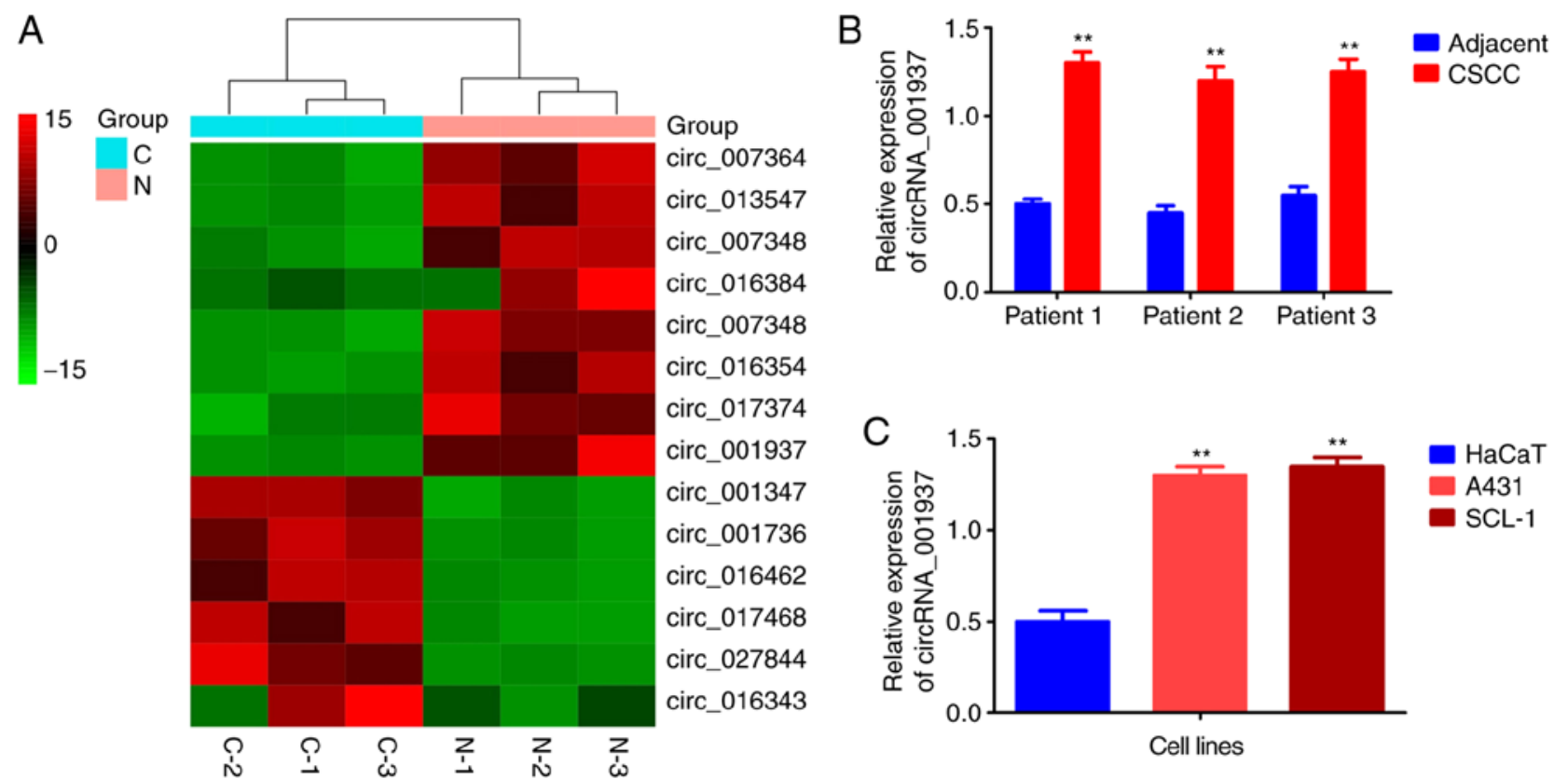

Figure 1. circRNA_001937 was upregulated in CSCC (n=3). (A) Cluster heat map of circRNA chip. (B) RT-qPCR assay revealed that circRNA_001937 was significantly increased in three pairs of CSCC tissues compared with corresponding adjacent tissues." $\mathrm{P}<0.01$ vs. adjacent tissues. (C) RT-qPCR assay revealed that circRNA_001937 was significantly increased in A431 and SCL-1 cells compared with HaCaT cell lines. ${ }^{* *} \mathrm{P}<0.01$ vs. HaCaT cell lines. N, adjacent normal tissues; C, CSCC tissues; CSCC, cutaneous squamous cell carcinoma; circRNA, circular RNA; RT-qPCR, reverse transcription-quantitative PCR.

A
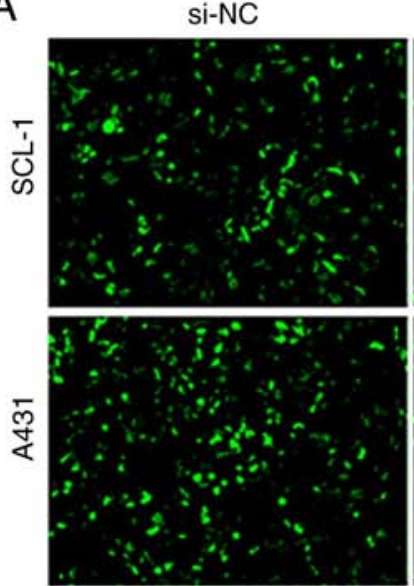

Si-circRNA_001937

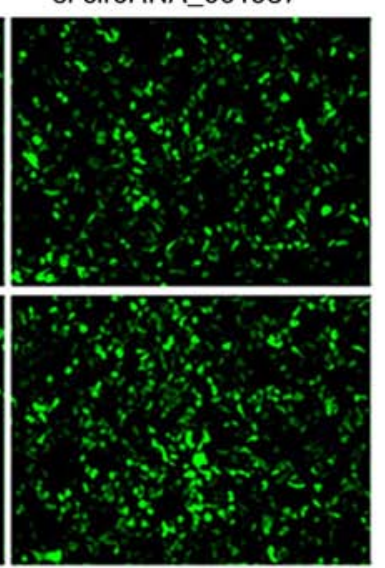

B

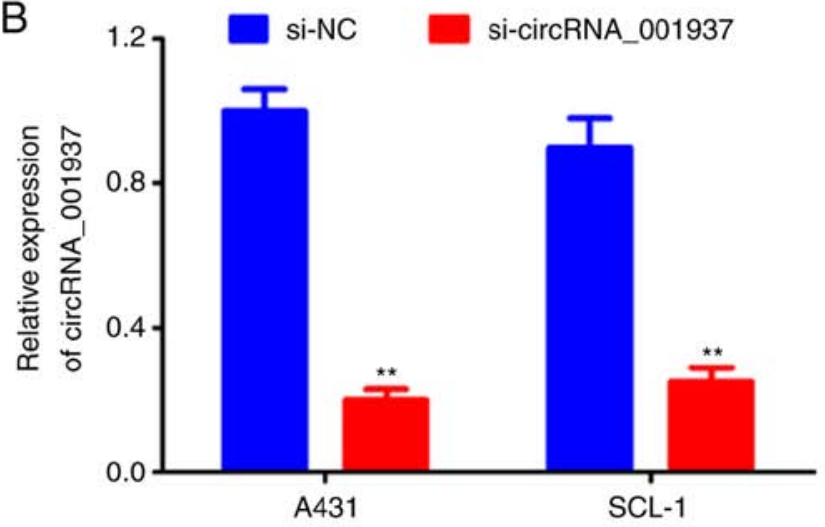

Figure 2. si-circRNA_001937 transfection. (A) Bright green fluorescence was observed under a confocal laser scanning microscope. (B) Reverse transcription-quantitative PCR results revealed that the expression of circRNA_001937 in the si-circRNA_001937 group were significantly lower compared with the si-NC group. ${ }^{* *} \mathrm{P}<0.01$ vs. si-NC group. siRNA/si, small interfering RNA; circRNA, circular RNA; NC, negative control.

A

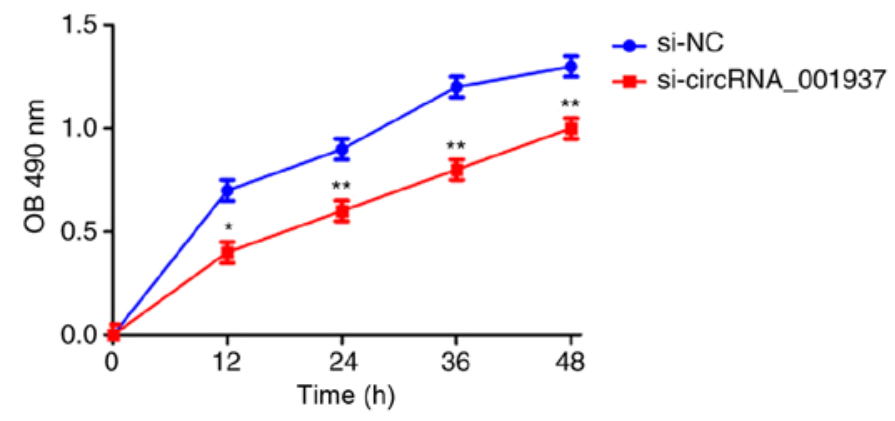

B

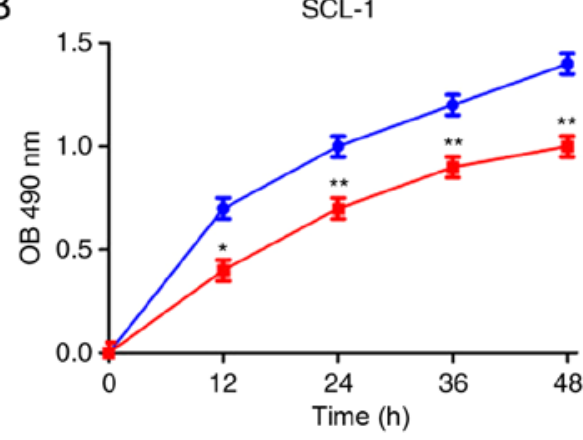

Figure 3. Silencing circRNA_001937 inhibits cell proliferation (n=3). (A) A431 cells. (B) SCL-1 cells. ${ }^{*} \mathrm{P}<0.05$ and ${ }^{* * *} \mathrm{P}<0.01$ vs. si-NC group. si, small interfering RNA; circRNA, circular RNA; OD, optical density; NC, negative control. 
A

A

$\bar{\alpha}$

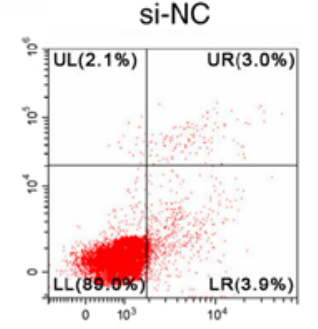

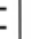

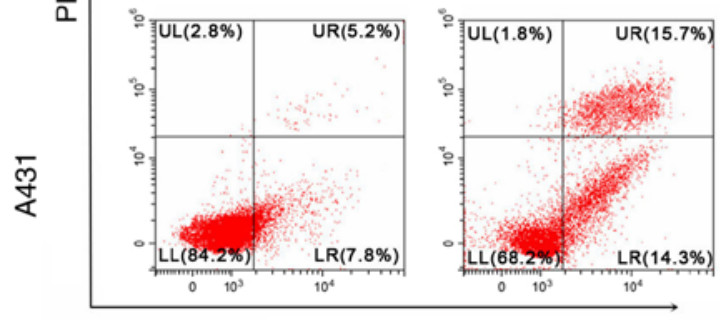

Annexin V-FITC
si-circRNA_001937

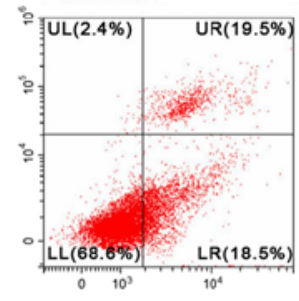

$10^{4}$
B

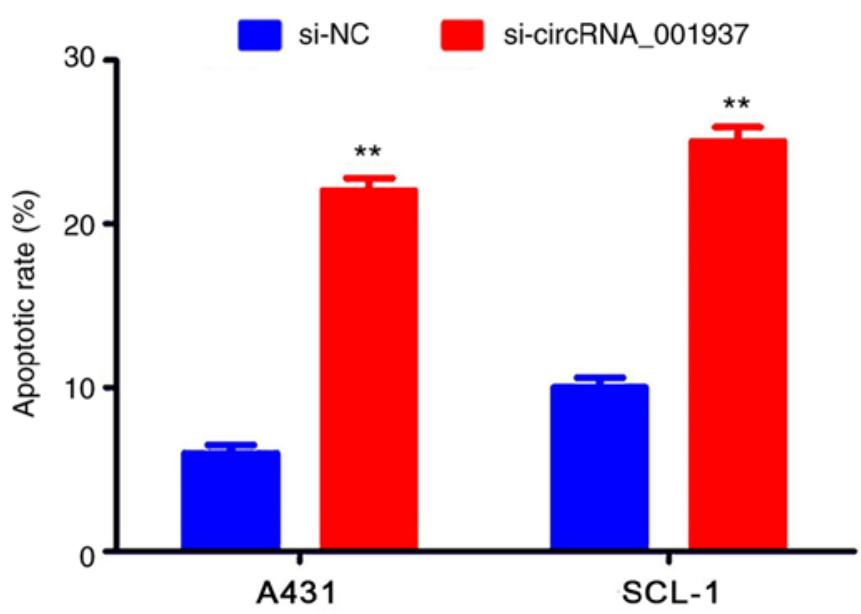

Figure 4. Silencing circRNA_001937 inhibits cell invasion and induces cell apoptosis ( $\mathrm{n}=3$ ). (A) Cell apoptosis rate determined using flow cytometry. (B) Analysis of the apoptotic rate. ${ }^{* *} \mathrm{P}<0.01$ vs. si-NC group. si, small interfering RNA; circRNA, circular RNA; NC, negative control; PI, propidium iodide.

A circRNA_001937 and miR-597-3p
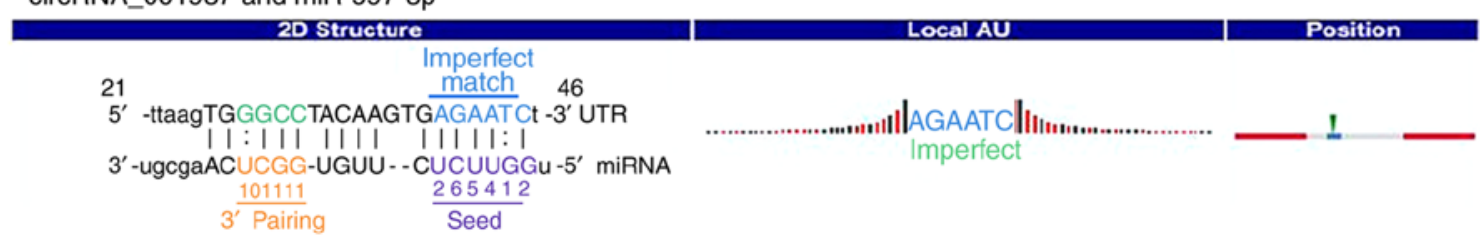

B
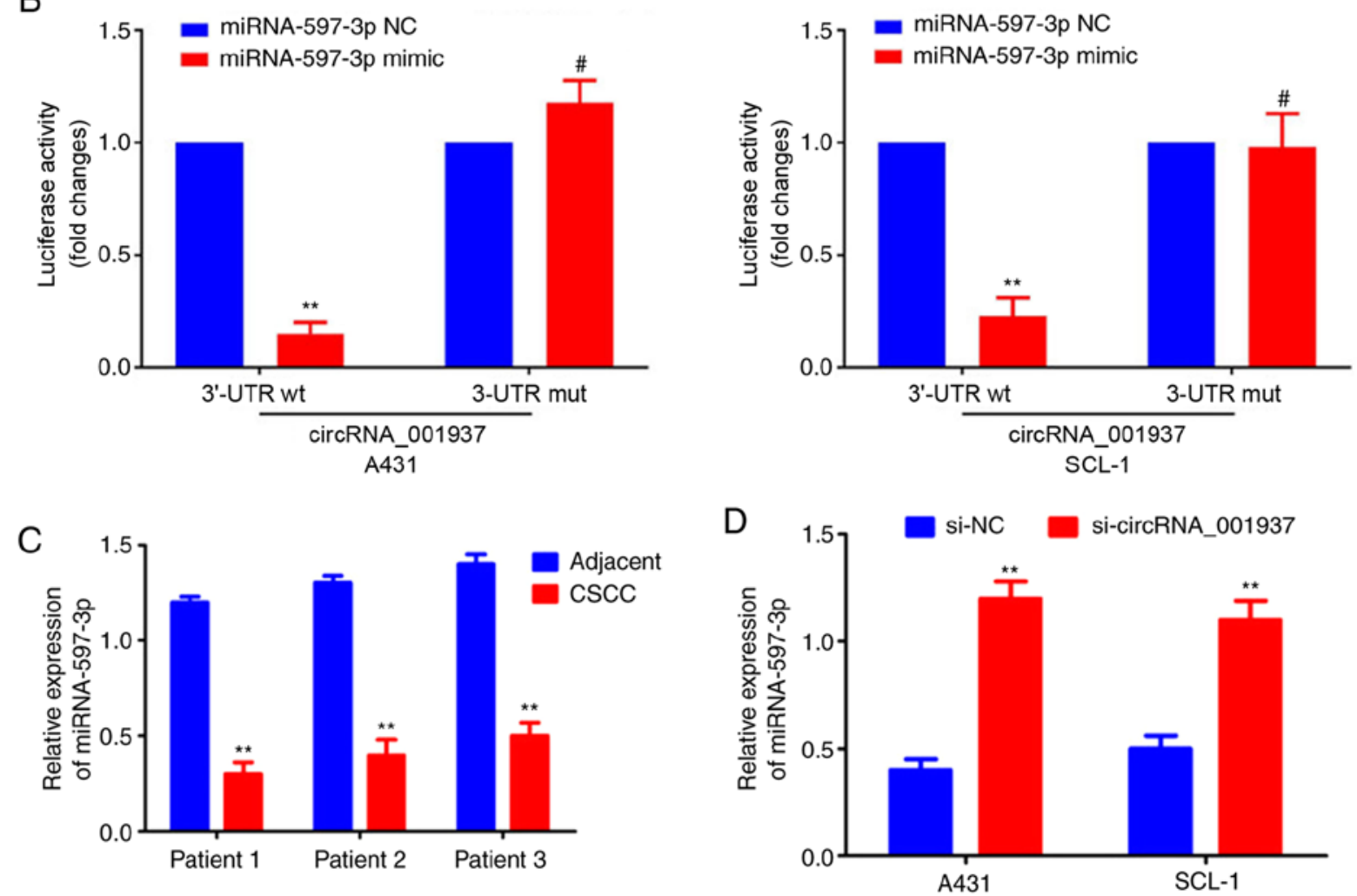

Figure 5. circRNA_001937 functions as an miRNA sponge for miRNA-597-3p. (A) Specific binding sequences of circRNA_001937 and miRNA-597-3p. (B) Dual-luciferase reporter assay results demonstrated the relative luciferase activity in each groups. ${ }^{*}$ "P $<0.01$ vs. circRNA_001937 wt + miRNA-597-3p NC; ${ }^{\text {"P }}<0.01$ vs. circRNA_001937 mut + miRNA-597-3p NC. (C) RT-qPCR results demonstrated that miRNA-597-3p was significantly downregulated in CSCC tissues compared with adjacent normal tissues. ${ }^{* *} \mathrm{P}<0.01$ vs. corresponding adjacent tissues. (D) RT-qPCR revealed that miRNA-597-3p was significantly increased following si-circRNA_001937 transfection. ${ }^{* *} \mathrm{P}<0.01$ vs. si-NC group. si-, small interfering RNA; circRNA, circular RNA; miRNA, microRNA; RT-qPCR, reverse transcription-quantitative PCR; CSCC, cutaneous squamous cell carcinoma; $\mathrm{NC}$, negative control; wt, wild type; mut, mutated type; UTR, untranslated region. 

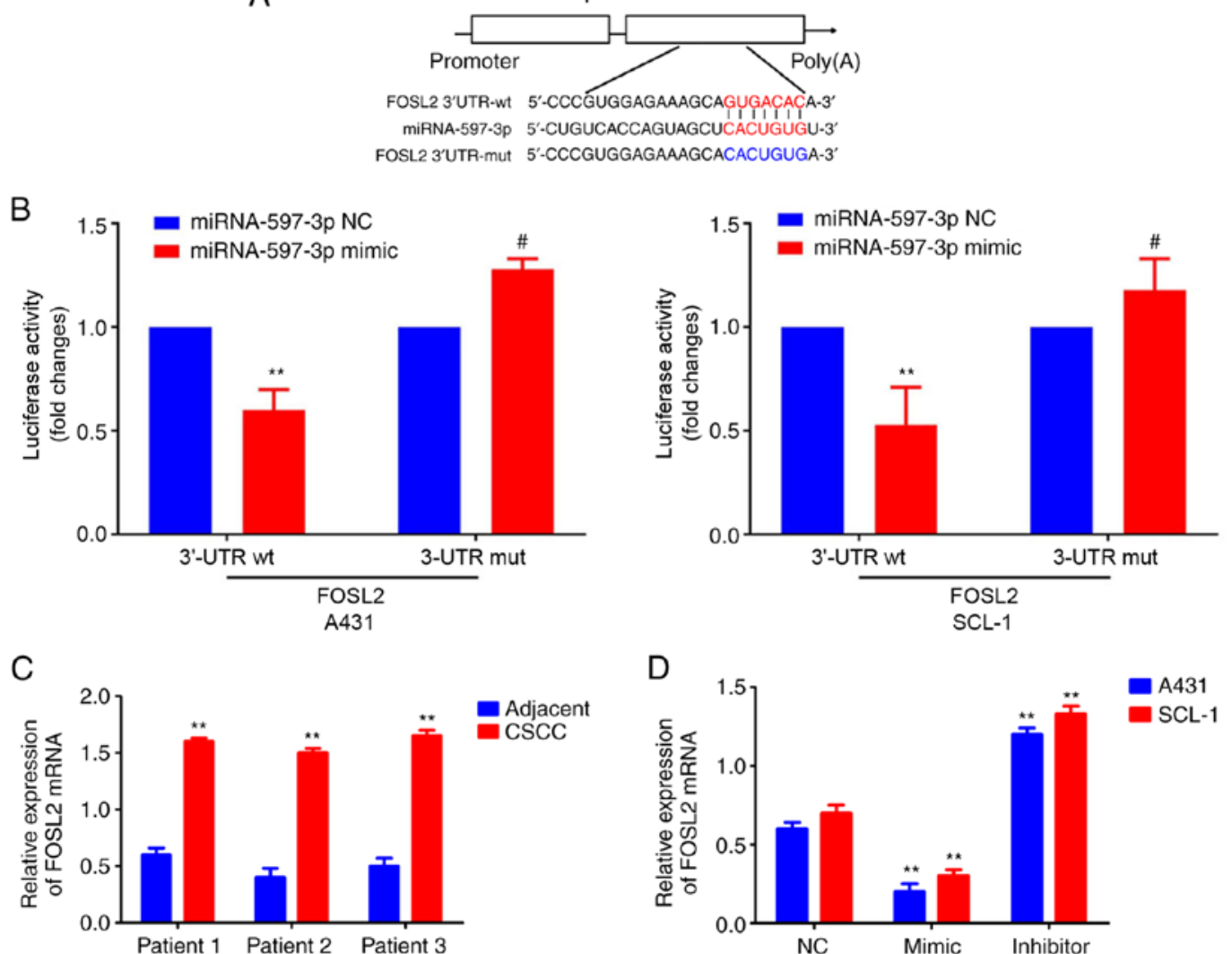

Figure 6. FOSL2 was identified as a direct target of miRNA-597-3p. (A) Specific binding sequences of FOSL2 3'-UTR and miRNA-597-3p were predicted using TargetScan 7.0. (B) Dual-luciferase reporter assay results demonstrated the relative luciferase activity in each group. ${ }^{* * *} \mathrm{P}<0.01 \mathrm{vs}$. FOSL2 wt + miRNA-597-3p NC; ${ }^{*} \mathrm{P}<0.01$ vs. FOSL2 mut + miRNA-597-3p NC. (C) Reverse transcription-quantitative PCR results demonstrated that FOSL2 mRNA was significantly upregulated in CSCC tissues. ${ }^{* *} \mathrm{P}<0.01$ vs. corresponding adjacent tissues. (D) FOSL2 mRNA were significantly decreased in a miRNA-597-3p mimic group, and substantially increased in miRNA-597-3p inhibitor group. ${ }^{* *} \mathrm{P}<0.01$ vs. miRNA-597-3p NC group. miRNA, microRNA; CSCC, cutaneous squamous cell carcinoma; NC, negative control; wt, wild type; mut, mutated type; UTR, untranslated region; FOSL2, Fos-related antigen 2.

were co-transfected with miRNA-597-3p mimics or the miRNA-597-3p NC and the wild-type (wt) or mutated (mut) 3'-untranslated region (UTR) of circRNA_001937 and FOSL2. Following $36 \mathrm{~h}$ of transfection, the luciferase activity was performed by a Dual-Luciferase Reporter Assay system (cat. no. E1910; Promega Corporation). Relative luciferase activity was normalized to the Renilla luciferase internal control.

Western blotting. A431 and SCL-1 cells of each group were lysed with RIPA buffer (Sigma-Aldrich; Merck KGaA). Protein quantification was carried out using a BCA protein assay kit (Promega Corporation). A total of $50 \mu \mathrm{g}$ protein/lane was separated by $10 \%$ SDS-PAGE and subsequently transferred to polyvinylidene difluoride membranes. The membranes were then blocked for $1 \mathrm{~h}$ at room temperature with non-fat dry milk in TBST (Bio-Rad Laboratories, Inc.). The membranes were incubated with the anti-FOSL2 primary antibody (1:500; cat. no. H00116173-B01P; Gibco; Thermo Fisher Scientific, Inc.) and anti- $\beta$-actin (1:1,000; cat. no. R2655; Sigma-Aldrich; Merck $\mathrm{KGaA})$ at $37^{\circ} \mathrm{C}$ overnight. Then, membranes were washed with PBS-Tween-20 buffer and subsequently incubated with a horseradish peroxidase-conjugated anti-rabbit antibody (1:1,000; cat. no. G-21234; Invitrogen; Thermo Fisher Scientific, Inc.) for $2 \mathrm{~h}$ at $4^{\circ} \mathrm{C}$. Protein bands were visualized using the Pierce ECL Western blotting substrate (Pierce; Thermo Fisher Scientific, Inc.).
Statistical analysis. Statistical analysis was performed using SPSS 17.0 software (SPSS, Inc.) and the data were presented as the mean \pm standard deviation. Statistical differences between two groups were determined using an unpaired Student's t-test or an $\chi^{2}$ test, whereas statistical differences between $>2$ groups were analyzed using a one-way analysis of variance, followed by Tukey's multiple comparison test. $\mathrm{P}<0.05$ was considered to indicate a statistically significant difference.

\section{Results}

circRNA_001937 expression levels are significantly increased in CSCC. The cluster heat map demonstrated that circRNA_001937 expression levels were increased by 14.58-fold in CSCC tissues (Fig. 1A). Similarly, results from the RT-qPCR also reported that circRNA_001937 levels were significantly increased in CSCC tissues $(\mathrm{P}<0.01$; Fig. 1B), and A431 and SCL-1 cells $(\mathrm{P}<0.01$; Fig. 1C) compared with the corresponding adjacent tissues and $\mathrm{HaCaT}$ cells.

si-circRNA_001937 transfection. Following cell transfection with si-circRNA_001937 or si-NC, bright green fluorescence was observed in the si-NC and si-circRNA_001937 groups (Fig. 2A). The expression levels of circRNA_001937 in the si-circRNA_001937 group were significantly decreased compared with the si-NC group ( $\mathrm{P}<0.01$; Fig. $2 \mathrm{~B})$. 

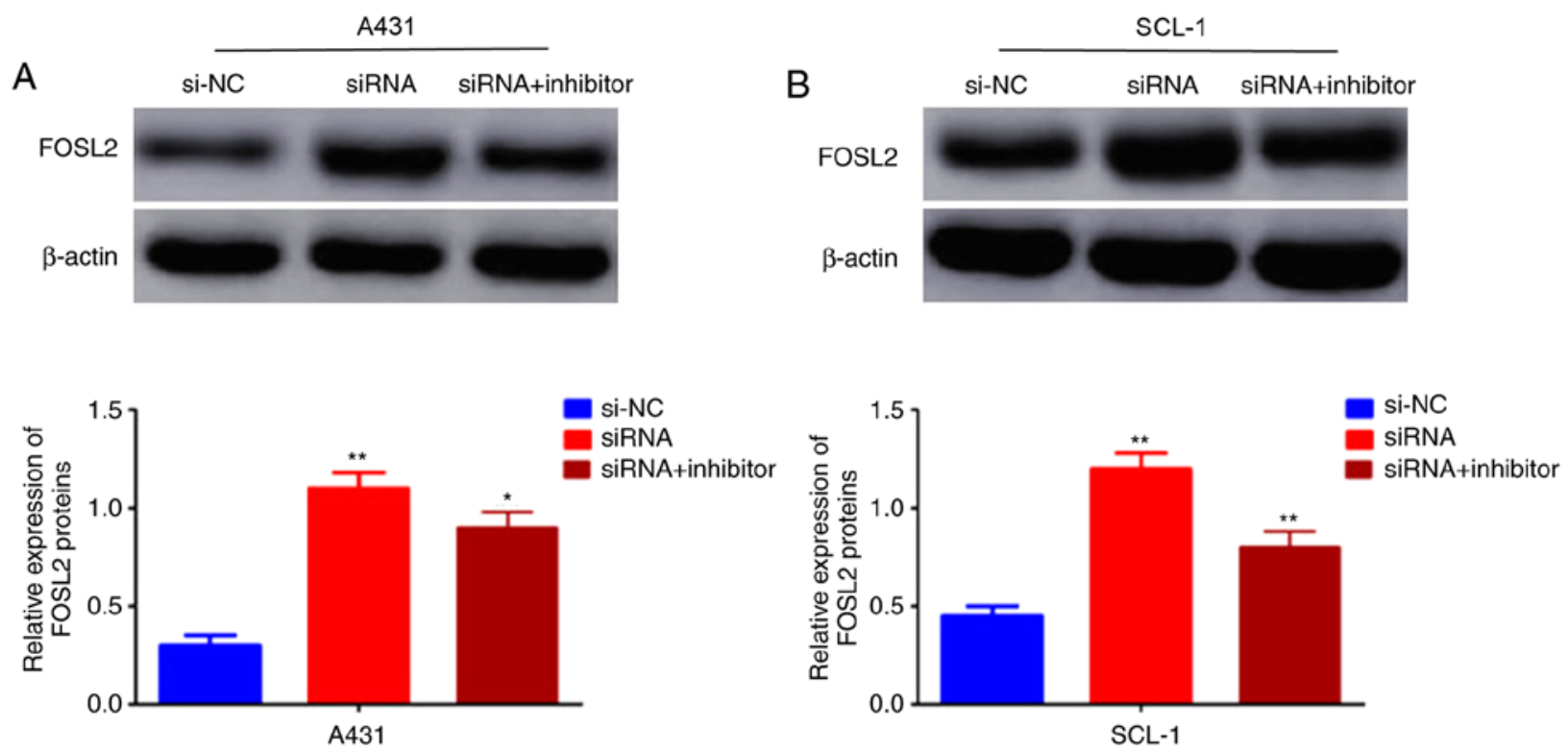

Figure 7. Effects of circRNA_001937/miRNA-597-3p axis on FOSL2. FOSL2 protein levels were examined in (A) A431 and (B) SCL-1 cells and were revealed to be significantly increased in the si-circRNA_001937 group, compared with the si-NC group, but substantially decreased in si-circRNA_001937 and miRNA-597-3p inhibitor co-transfection group, compared with the si-circRNA_001937 group. " $\mathrm{P}<0.05$ and ${ }^{* *} \mathrm{P}<0.01$ vs. control group. circRNA, circular RNA; siRNA/si-, small interfering RNA; miRNA, microRNA; FOSL2, Fos-related antigen 2; NC, negative control.

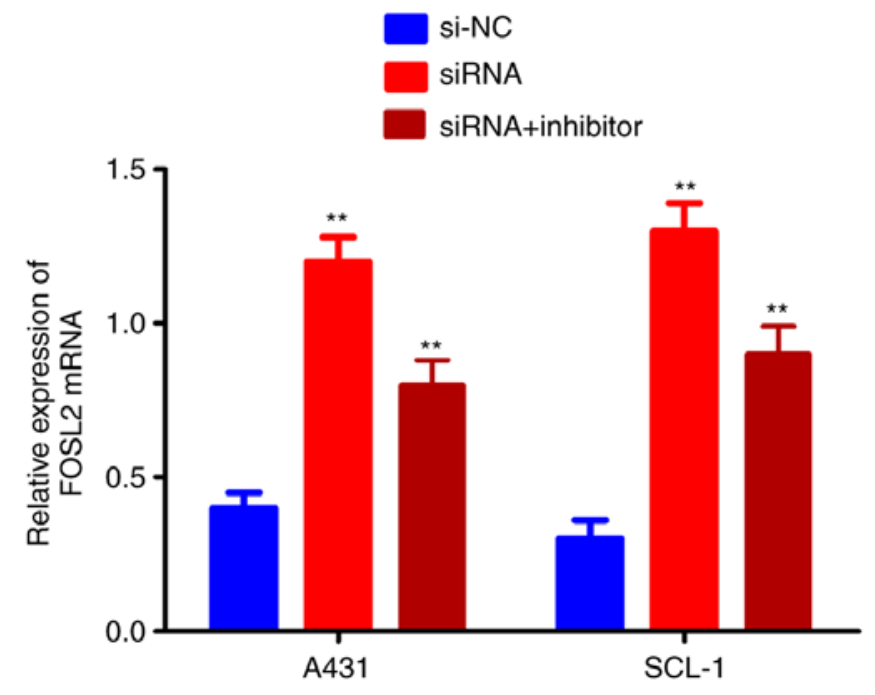

Figure 8. Effect of circRNA_001937/miRNA-597-3p axis on FOSL2. FOSL2 mRNA levels were significantly increased in the si-circRNA_001937 group compared with the si-NC group, but substantially decreased in the si-circRNA_001937 and miRNA-597-3p inhibitor co-transfection group compared with the si-circRNA_001937 group. ${ }^{* *} \mathrm{P}<0.01$ vs. control group. circRNA, circular RNA; siRNA/si-, small interfering RNA; miRNA, microRNA; FOSL2, Fos-related antigen 2; NC, negative control; CSCC, cutaneous squamous cell carcinoma.

Silencing circRNA_001937 expression inhibits CSCC proliferation, and induces apoptosis. Subsequently, the effect of circRNA_001937 on cell behavior was investigated. The proliferative rate $(\mathrm{P}<0.05)$ were significantly reduced in the si-circRNA_001937 group compared with the si-NC group (Fig. 3). In addition, flow cytometric analysis identified that the apoptotic rate was significantly increased in the si-circRNA_001937 group compared with the si-NC group (P<0.01; Fig. 4).
circRNA_001937 functions as an miRNA sponge for miRNA-597-3p. The specific binding sequences between circRNA_001937 and miRNA-597-3p are presented in Fig. 5A. Dual-luciferase reporterassays were used to demonstrate that the relative luciferase activity in the circRNA_001937-3'-UTR-Wt and miRNA-597-3p mimic co-transfection group was significantly decreased compared with the groups co-transfected with miRNA-597-3p NC or circRNA_001937-3'-UTR-Mut ( $\mathrm{P}<0.01$; Fig. 5B). miRNA-597-3p expression levels were also significantly decreased in CSCC tissues compared with corresponding adjacent tissues $(\mathrm{P}<0.01$; Fig. $5 \mathrm{C})$; however, these miRNA-597-3p expression levels were significantly increased following transfection with si-circRNA_001937 compared with the si-NC group ( $\mathrm{P}<0.01$; Fig. 5D).

FOSL2 is a direct target of miRNA-597-3p. The specific binding sequences between FOSL2 3'-UTR and miRNA-597-3p were predicted using TargetScan 7.0 software (Fig. 6A). Dual-luciferase reporter assay results demonstrated that the relative luciferase activity in cells co-transfected with FOSL2-3'-UTR-Wt and miRNA-597-3p mimic was significantly decreased compared with the miRNA-597-3p NC or FOSL2-3'-UTR-Mut groups ( $\mathrm{P}<0.01$; Fig. 6B). In addition, FOSL2 mRNA expression levels were significantly increased in CSCC tissues compared with the adjacent noncancerous tissues $(\mathrm{P}<0.01$; Fig. 6C), whereas FOSL2 expression levels were significantly decreased in the miRNA-597-3p mimic group, and significantly increased in the miRNA-597-3p inhibitor group, compared with the miRNA-597-3p NC group (P<0.01; Fig. 6D).

FOSL2 is activated by the circRNA_001937/miRNA-597-3p axis. The effects of the circRNA_001937/miRNA-597-3p axis on FOSL2 mRNA and protein expression levels was further investigated using RT-qPCR and western blotting, respectively. The expression levels of FOSL2 protein $(\mathrm{P}<0.01$; Fig. 7A and $\mathrm{B})$ 
and mRNA ( $\mathrm{P}<0.01$; Fig. 8) were significantly increased in the si-circRNA_001937 group compared with the si-NC group; and were substantially decreased in the si-circRNA_001937 and miRNA-597-3p inhibitor co-transfection group compared with the si-circRNA_001937 group.

\section{Discussion}

circRNAs serve as gene regulators in a variety of physiological functions and pathological processes, of which their functions in numerous types of cancer are currently being investigated. For example, circ_0003159 was identified as a potential biomarker for patients with gastric cancer. circ_001569 was identified to serve as a sponge for miR-145, which may prove beneficial as increased miR-145 expression promotes proliferation and invasion in colorectal cancer (14). Furthermore, circ_0043278 promoted non-small cell lung cancer (NSCLC) proliferation and migration through regulating miR-520 expression (15). Previous studies have also reported that circ_0016788 regulated hepatocellular carcinoma (HCC) tumorigenesis through the miR-486/cyclin-dependent kinase 4 pathway (16-18) and circRNA-mitochondrial tRNA translation optimization 1, whose expression was decreased in $\mathrm{HCC}$, and was observed to sequester miRNA-9 and suppress HCC progression.

In the present study, the circRNAs chip and RT-qPCR results demonstrated that circRNA_001937 expression levels were increased in CSCC, and that silencing circRNA_001937 inhibited cell proliferation and invasion and induced cell apoptosis. circRNA_001937 is an exonic circRNA that is 2,850 nucleotides in length, and is located on chromosome 16 (19). Huang et al (20) reported that circRNA_001937 expression was significantly increased in the peripheral blood mononuclear cells of patients with tuberculosis, and that circRNA_001937 was correlated with tuberculosis severity, with expression levels successfully decreasing following treatment The results obtained in the present study suggest that circRNA_001937 may be used as a potential diagnostic biomarker for CSCC. However, the number of patients with CSCC used in the present study was small, and large-scale clinical samples and adequate follow-up studies are required for further verification. In addition, dual-luciferase reporter assays confirmed that circRNA_001937 served as a miRNA sponge towards miRNA-597-3p, which subsequently increased FOSL2 expression. There are a limited number of studies examining the miRNA-597-3p/FOSL2 pathway. miRNA-597-3p is located on the 8p23.1 chromosome (21). Xie et al (22) reported that miR-597 targeting 14-3-30 enhances cellular invasion and the epithelial-mesenchymal transition in nasopharyngeal carcinoma cells, whilst Zhang et al (23) revealed that a low expression of miR-597 is correlated with tumor stage and a poor outcome in breast cancer. FOSL2 is a member of the AP-1 transcription factor family (24). Previous studies have demonstrated that FOSL2 is abnormally expressed in numerous different types of tumor. Wang et al (25) identified that FOSL2 may positively regulate transforming growth factor- $\beta 1$ signaling in NSCLC. Sun et al (26) confirmed that miR-143-3p inhibited the proliferation, migration and invasion of osteosarcoma through targeting FOSL2. Additionally, FOSL2 expression may be regulated through a number of different mechanisms. miRNA regulation is one method, including miRNA-30e (27) and miR-143-3p (26), which have been revealed to regulate the expression of FOSL2. These studies demonstrate that miRNA-597-3p and FOSL2 participate in the carcinogenesis and development of cancer. The present study demonstrated that miRNA-597-3p expression was significantly decreased, and FOSL2 expression was significantly increased, in CSCC tissues. The FOSL2 gene was additionally observed to be directly targeted by miRNA-597-3p, and FOSL 2 expression levels were observed to be increased by circRNA_001937 serving as a sponge for miRNA-597-3p.

In conclusion, to the best of our knowledge, the present study provides the first evidence that circRNA_001937 expression is significantly increased in CSCC, and that silencing circRNA_001937 inhibits CSCC proliferation and invasion and induces apoptosis. Silencing circRNA_001937 gene expression may inhibit CSCC progression by preventing the sponging of the miRNA-597-3p/FOSL2 pathway. These results suggest a novel, potential therapeutic target for the treatment of patients with CSCC. Large-scale, clinical and adequate follow-up studies are required for further verification of these results.

\section{Acknowledgements}

Not applicable.

\section{Funding}

No funding was received.

\section{Availability of data and materials}

The datasets used and/or analyzed during the current study are available from the corresponding author on reasonable request.

\section{Authors' contributions}

LG and QL designed the study and performed the experiments. HJJ and DZ analyzed the data. All authors have read and approved the final manuscript.

\section{Ethics approval and consent to participate}

The present study was approved by the Ethics Committee of the First Hospital of Jilin University (Jilin, China), and written informed consent was obtained from all patients.

\section{Patient consent for publication}

Not applicable.

\section{Competing interests}

The authors declare that they have no competing interests.

\section{References}

1. Castelo B, Viñal D, Maseda R, Ostios L, Sánchez D, García-Salvatierra B, Escámez MJ, Martínez-Santamaría L, Del Río M, Mora-Rillo M, et al: Epidemiology and natural history of cutaneous squamous cell carcinoma in recessive dystrophic epidermolysis bullosa patients: 20 years' experience of a reference centre in Spain. Clin Transl Oncol 21: 1573-1577, 2019. 
2. Xiong Y, Dresser K and Cornejo KM: Frequent TLE1 expression in cutaneous neoplasms. Am J Dermatopathol 41: 1-6, 2019.

3. Ogata D and Tsuchida T: Systemic immunotherapy for advanced cutaneous squamous cell carcinoma. Curr Treat Options Oncol 20: 30, 2019.

4. Ding L, Zhao Y, Dang S, Wang Y, Li X, Yu X, Li Z, Wei J, Liu M and Li G: Circular RNA circ-DONSON facilitates gastric cancer growth and invasion via NURF complex dependent activation of transcription factor SOX4. Mol Cancer 18: 45, 2019.

5. Li Q, Deng C, Zhu T, Ling J, Zhang H, Kong L, Zhang S, Wang J and Chen X: Dynamics of physiological and miRNA changes after long-term proliferation in somatic embryogenesis of Picea balfouriana. Trees 33: 469-480, 2019.

6. Niwa Y, Yamada S, Sonohara F, Kurimoto K, Hayashi M, Tashiro M, Iwata N, Kanda M, Tanaka C, Kobayashi D, et al: Identification of a serum-based miRNA signature for response of esophageal squamous cell carcinoma to neoadjuvant chemotherapy. J Transl Med 17: 1, 2019.

7. Kogure A, Kosaka N and Ochiya T: Cross-talk between cancer cells and their neighbors via miRNA in extracellular vesicles: An emerging player in cancer metastasis. J Biomed Sci 26: 7, 2019.

8. He J, Mai J, Li Y, Chen L, Xu H, Zhu X and Pan Q: miR-597 inhibits breast cancer cell proliferation, migration and invasion through FOSL2. Oncol Rep 37: 2672-2678, 2017.

9. Wrann CD, Eguchi J, Bozec A, Xu Z, Mikkelsen T, Gimble J, Nave H, Wagner EF, Ong SE and Rosen ED: FOSL2 promotes leptin gene expression in human and mouse adipocytes. J Clin Invest 122: 1010-1021, 2012.

10. Jahangiri L, Sharpe M, Novikov N, González-Rosa JM, Borikova A, Nevis K, Paffett-Lugassy N, Zhao L, Adams M, Guner-Ataman B, et al: The AP-1 transcription factor component Fosl2 potentiates the rate of myocardial differentiation from the zebrafish second heart field. Development 143: 113-122, 2016.

11. Hu Y, Li J, Li S, Wang S, Gao G and Hou J: Expression level of FOSL2 mRNA in blood leukocytes of type 2 diabetes mellitus patients in Uygurs in Xinjiang and its clinical sinificance. J Jilin Univ 42: 545-550, 2016 (In Chinese).

12. Li Z, Liu Y, Yan J, Zeng Q, Hu Y, Wang H, Li H, Li J and Yu Z: Circular RNA hsa_circ_0056836 functions an oncogenic gene in hepatocellular carcinoma through modulating miR-766-3p/FOSL2 axis. Aging (Albany NY) 12: 2485-2497, 2020.

13. Livak KJ and Schmittgen TD: Analysis of relative gene expression data using real-time quantitative PCR and the 2(-Delta Delta C(T)) method. Methods 25: 402-408, 2001.

14. Xie H, Ren X, Xin S, Lan X, Lu G, Lin Y, Yang S, Zeng Z, Liao W, Ding YQ and Liang L: Emerging roles of circRNA_001569 targeting miR-145 in the proliferation and invasion of colorectal cancer. Oncotarget 7: 26680-26691, 2016.

15. Cui J, Li W, Liu G, Chen X, Gao X, Lu H and Lin D: A novel circular RNA, hsa_circ 0043278, acts as a potential biomarker and promotes non-small cell lung cancer cell proliferation and migration by regulating miR-520. Artif Cells Nanomed Biotechnol 47: 810-821, 2019.
16. Guan Z, Tan J, Gao W, Li X, Yang Y, Li X, Li Y and Wang Q: Circular RNA hsa_circ_0016788 regulates hepatocellular carcinoma tumorigenesis through miR-486/CDK4 pathway. J Cell Physiol 234: 500-508, 2018.

17. Han D, Li J, Wang H, Su X, Hou J, Gu Y, Qian C, Lin Y, Liu X, Huang M, et al: Circular RNA circMTO1 acts as the sponge of microRNA-9 to suppress hepatocellular carcinoma progression. Hepatology 66: 1151-1164, 2017.

18. Zhou J, Zhang WW, Peng F, Sun JY, He ZY and Wu SG: Downregulation of hsa_circ_0011946 suppresses the migration and invasion of the breast cancer cell line MCF-7 by targeting RFC3. Cancer Manag Res 10: 535-544, 2018.

19. Ojha R, Nandani R, Chatterjee N and Prajapati VK: Emerging role of circular RNAs as potential biomarkers for the diagnosis of human diseases. Adv Exp Med Biol 1087: 141-157, 2018.

20. Huang ZK, Yao FY, Xu JQ, Deng Z, Su RG, Peng YP, Luo Q and Li JM: Microarray expression profile of circular RNAs in peripheral blood mononuclear cells from active tuberculosis patients. Cell Physiol Biochem 45: 1230-1240, 2018.

21. Bay A, Coskun E, Oztuzcu S, Ergun S, Yilmaz F and Aktekin E: Plasma microRNA profiling of pediatric patients with immune thrombocytopenic purpura. Blood Coagul Fibrinolysis 25: 379-383, 2014.

22. Xie L, Jiang T, Cheng A, Zhang T, Huang P, Li P, Wen G, Lei F, Huang Y, Tang X, et al: MiR-597 targeting 14-3-30 enhances cellular invasion and EMT in nasopharyngeal carcinoma cells. Curr Mol Pharmacol 12: 105-114, 2019.

23. Zhang XY, Liu DJ, Yuan RB, Zhang DH, Li SR, Zhang SH and Zhang LY: Low expression of miR-597 is correlated with tumor stage and poor outcome in breast cancer. Eur Rev Med Pharmacol Sci 22: 456-460, 2018.

24. Li S, Fang XD, Wang XY and Fei BY: Fos-like antigen 2 (FOSL2) promotes metastasis in colon cancer. Exp Cell Res 373: $57-61,2018$

25. Wang J, Sun D, Wang Y, Ren F, Pang S, Wang D and Xu S: FOSL2 positively regulates TGF- $\beta 1$ signalling in non-small cell lung cancer. PLoS One 9: e112150, 2014.

26. Sun X, Dai G, Yu L, Hu Q, Chen J and Guo W: miR-143-3p inhibits the proliferation, migration and invasion in osteosarcoma by targeting FOSL2. Sci Rep 8: 606, 2018

27. Ling L, Zhang SH, Zhi LD, Li H, Wen QK, Li G and Zhang WJ: MicroRNA-30e promotes hepatocyte proliferation and inhibits apoptosis in cecal ligation and puncture-induced sepsis through the JAK/STAT signaling pathway by binding to FOSL2. Biomed Pharmacother 104: 411-419, 2018.

This work is licensed under a Creative Commons Attribution-NonCommercial-NoDerivatives 4.0 International (CC BY-NC-ND 4.0) License. 